\title{
A Novel Mutation in the TBG Gene Producing Partial Thyroxine-Binding Globulin Deficiency (Glencoe) Identified in 2 Families
}

\author{
Theodora Pappa ${ }^{a} \quad$ Lars C. Moeller ${ }^{\mathrm{e}}$ Deborah V. Edidin ${ }^{d}$ Silvana Pannain ${ }^{\mathrm{a}}$ \\ Samuel Refetoff ${ }^{a-c}$
}

Departments of a Medicine and ${ }^{b}$ Pediatrics, and ${ }^{c}$ Committee on Genetics, The University of Chicago, and ${ }^{d}$ Department

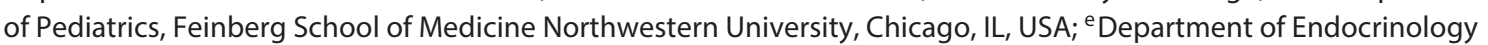
and Division of Laboratory Research, University Hospital Essen, University of Duisburg-Essen, Essen, Germany

\section{What Is Known about This Topic?}

- Partial thyroxine-binding globulin deficiency (TBG-PD) is an X-linked inherited TBG defect, which produces alterations in thyroid function tests, but no metabolic abnormality.

- Clinical awareness is needed to correctly diagnose affected individuals and avoid unnecessary treatment.

- Genetic testing is valuable in identifying mutation carriers and providing genetic counseling.

\section{What Does This Case Report Add?}

- The study of these 2 cases with TBG-PD identified a novel TBG variant that was not reported previously.

- Further evaluation of the molecular properties of the mutant molecule with isoelectric focusing and $\mathrm{T}_{4}$ binding studies in cell culture demonstrated that the mutant TBG has normal protein isoforms but decreased affinity to bind thyroid hormone.

\section{Keywords}

Mutation - Thyroxine-binding globulin · Thyroxine-binding globulin deficiency $\cdot \mathrm{T}_{4}$-binding affinity

\section{Abstract \\ Background: Thyroxine-binding globulin (TBG) is the major thyroid hormone transport protein in serum. Located on the long arm of the $\mathrm{X}$ chromosome, TBG (SERPINA7) gene muta- tions most commonly produce inherited partial TBG defi- ciency (TBG-PD). Objective: We report a novel TBG variant associated with TBG-PD identified in 2 different families of}

Ashkenazi origin residing in greater Chicago. Methods: Family 1 : The proband was 12.6 years old when she presented for delayed puberty and was placed on $\mathrm{L}_{-} \mathrm{T}_{4}$. Although her serum TSH normalized, her serum $\mathrm{T}_{4}$ remained low. Affected family members had low total $T_{4}$ and $T_{3}$, but a normal free $T_{4}$ index, even when serum TSH concentrations were normal. Family 2: A 71-year-old male presented with a history of a nonfunctioning pituitary adenoma and normal pituitary axes except for low total $\mathrm{T}_{4}$ and $\mathrm{T}_{3}$. His brother had a similar

T.P. and L.C.M. contributed equally to this work.

\section{KARGER}

E-Mail karger@karger.com www.karger.com/etj 
thyroid phenotype. Results: Following direct DNA sequencing, both index patients were found to carry a missense mutation in the TBG gene (c.751T>G) producing p.V215G. The proposita of family 1 was heterozygous and the proband in family 2 was hemizygous for the mutation. Isoelectric focusing showed no alteration in the TBG isoforms and in vitro expression demonstrated a TBG with reduced affinity for $T_{4}$. Conclusions: We report a novel mutation in the TBG gene in 2 unrelated families that produces a molecule with reduced affinity for $\mathrm{T}_{4}$ resulting in low serum $\mathrm{T}_{4}$. However, the physical properties of the mutant molecule remained unaltered as determined by isoelectric focusing.

(C) 2017 European Thyroid Association Published by S. Karger AG, Basel

\section{Introduction}

Thyroxine-binding globulin (TBG) is one of the major serum transport proteins for iodothyronines, with the other two being transthyretin and human serum albu$\mathrm{min}$. TBG is a glycoprotein with a $54-\mathrm{kDa}$ single polypeptide chain synthesized in the liver. The mature molecule consists of 395 amino acids and 4 heterosaccharide units containing 5-9 terminal sialic acids, which are important for appropriate posttranslational folding and secretion of the molecule. TBG is encoded by 1 gene copy on the long arm of the X chromosome (Xq22.2). The gene has 5 exons, 4 of which are coding, and 2 polyadenylation sites resulting in 2 different mRNA transcripts $[1,2]$.

Inherited TBG defects, i.e. complete and partial TBG deficiency and TBG excess, follow an X-linked pattern; they do not cause disease or affect the metabolic status, but they produce alterations in the thyroid function tests (TFTs). Patients typically present with euthyroid hypoor hyperthyroxinemia, which may not be readily recognized and thus lead to inappropriate treatment with its associated side effects.

Partial TBG deficiency (TBG-PD) is the most common form of inherited TBG deficiency with a frequency of 1 in 4,000 newborns. Affected males present with the full spectrum of TBG deficiency, which consists of low total $\mathrm{T}_{4}$ and $\mathrm{T}_{3}$ and low but detectable TBG levels in the serum, whereas free TH levels are normal. In heterozygous females, TBG levels are usually above half the normal TBG concentration, intermediate between affected and unaffected males, because of the random inactivation of 1 of the $2 \mathrm{X}$ chromosomes. All TBG-PD variants reported so far were caused by missense mutations [3]. Here we report 2 families harboring a novel TBG variant: V215G or TBG-Glencoe.

\section{Patients and Methods}

Case History

Family 1: The proposita was 12.6 years old when she presented for delayed puberty and was placed on $\mathrm{L}_{-} \mathrm{T}_{4}$ for "generous $\mathrm{TSH}$ " (value unknown). Although her TSH normalized, her serum $\mathrm{T}_{4}$ remained low. Her development was normal. At 17 years of age and after discontinuing treatment with $\mathrm{L}_{-} \mathrm{T}_{4}$, her total serum $\mathrm{T}_{4}$ $\left(\mathrm{TT}_{4}\right)$ and $\mathrm{T}_{3}\left(\mathrm{TT}_{3}\right)$ levels were low, as was her $\mathrm{TBG}$ at $1.4 \mathrm{mg} / \mathrm{dL}$ (normal 2.0-5.3). Her free $\mathrm{T}_{4}$ index $\left(\mathrm{FT}_{4} \mathrm{I}\right)$ was low-normal and TSH level was elevated allegedly due to autoimmune hypothyroidism (Fig. 1a). No signs or symptoms of hypothyroidism were present. Her father and younger sister also had low $\mathrm{TT}_{4}$ and $\mathrm{TT}_{3}$, but normal $\mathrm{FT}_{4} \mathrm{I}$ and TSH concentrations. The TFTs of her mother and 2 younger brothers were within the normal range.

Family 2: The proband, a 71-year-old male, presented with a history of a stable nonfunctioning pituitary adenoma. Testing of his pituitary axis was normal except for low testosterone, $\mathrm{TT}_{4}$, and $\mathrm{TT}_{3}$. Total reverse $\mathrm{T}_{3}\left(\mathrm{TrT}_{3}\right)$ levels and TBG of $1.0 \mathrm{mg} / \mathrm{dL}$ were also low, whereas $\mathrm{FT}_{4}$ by equilibrium dialysis of $1.2 \mathrm{ng} / \mathrm{dL}$ (normal range $0.8-2.0$ ) and TSH were normal (Fig. 1b). His brother had a similar thyroid phenotype. Unfortunately, no other family member gave consent to be tested.

Both index subjects were clinically euthyroid and nonconsanguineous, and both families were Ashkenazi Jews currently residing in the greater Chicago area, whose ancestors originated from Russia. The combination of their clinical picture and alterations in TFTs in the probands and their family members raised the suspicion of TBG-PD.

All subjects of the study gave their informed consent prior to testing. The study protocol was approved by the University of Chicago committee on human research.

Thyroid Function Tests

We measured the serum total thyroid hormone concentration $\left(\mathrm{TT}_{4}, \mathrm{TT}_{3}\right.$, and $\mathrm{Tr}_{3}$ ), $\mathrm{TSH}$, thyroglobulin (TG), and antibodies to TG and thyroperoxidase. Free $\mathrm{T}_{4}$ was measured by equilibrium dialysis and TBG by an immunometric assay with a sensitivity of $3.0-3.5 \mu \mathrm{g} / \mathrm{mL}(0.055-0.065 \mu \mathrm{mol} / \mathrm{L})[4]$.

\section{DNA Mutation Analysis}

Genomic DNA was isolated from samples of peripheral blood leukocytes using the QIAamp DNA Mini Kit (QIAGEN, Valencia, CA, USA) followed by PCR amplification and direct sequencing [5].

\section{Isoelectric Focusing}

Samples mixed with buffer containing $\left[{ }^{125} \mathrm{I}\right]-\mathrm{T}_{4}$ were electrophoresed at $5{ }^{\circ} \mathrm{C}$ on ampholine-containing gels, which were subsequently dried and autoradiographed [4]. A sample from a hemizygous male (I-1 of family 1 ) and a heterozygous female (II-2 of family 1) were compared with serum samples from an unaffected family member, an external control individual, a normal female on estrogen therapy and a pregnant individual.

\section{Binding Studies}

Cos-7 cells were cultured in Dulbecco's modified Eagle's medium (DMEM; Gibco) supplemented with 10\% fetal bovine serum and $1 \%$ penicillin-streptomycin (Gibco). The cells were transiently transfected to express either the normal TBG or the V215G variant. Binding of $\mathrm{T}_{4}$ to the protein secreted into the medium was measured [6]. 

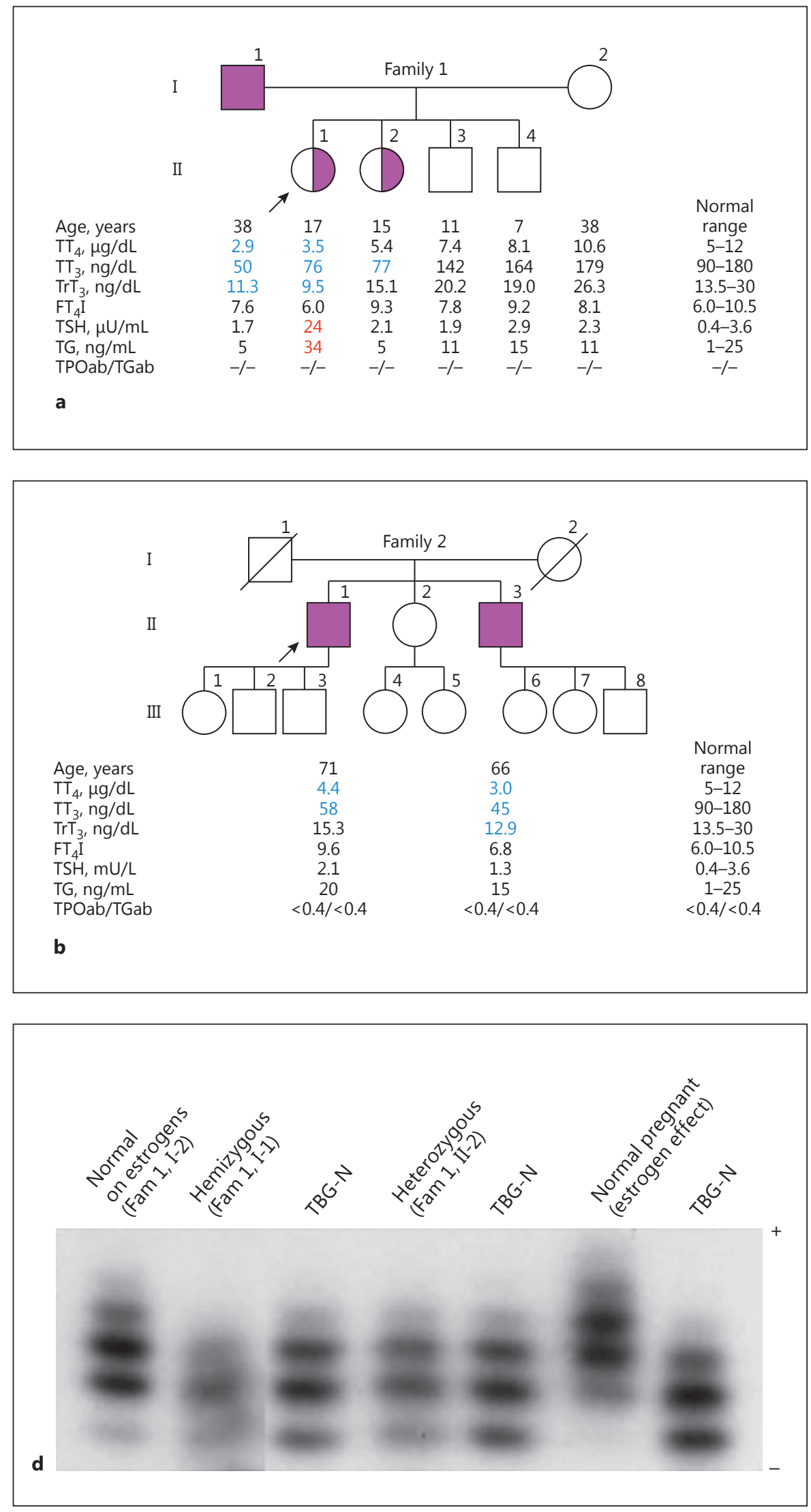

Fig. 1. a Pedigree of family 1: TFT results are aligned with each symbol representing a family member. Abnormally high values are in red and low values in blue (colors refer to the online version only). The arrow denotes the proband. Filled symbols are affected hemizygous and half-filled symbols are heterozygous. b Pedigree of family 2, presented in the same way as family 1 . The serum TBG concentrations for the probands of families 1 and 2 are in the text. c Sequencing chromatograms corresponding to a normal individual and a hemizygous and heterozygous individual with the V215G TBG variant. d IEF gel showing the TBG isoform patterns in serum from a hemizygous and a heterozygous patient compared to a normal family member, a normal control (TBG-N), a pregnant individual and an individual on estrogens. TPO, thyroperoxidase.

Pappa/Moeller/Edidin/Pannain/Refetoff 


\section{Results}

The probands of both families were found to harbor a missense mutation in the TBG gene, namely a single nucleotide substitution in exon 2 ( $\mathrm{T}$ to $\mathrm{G}$ ), codon 215 . This mutation resulted in the replacement of the normal valine (GTG) with a glycine (GGG) (V215G, 1 being the first amino acid of the mature TBG molecule; Fig. 1c).

The proposita of family 1 , as well as her younger sister, were heterozygous and their father hemizygous for the V215G variant, whereas the other family members were normal. The proband of family 2 and his brother were both hemizygous for the same TBG variant. The mother of the proband (I-2) and the daughters of the proband (III-1) and his brother (III-6 and III-7) are obligatory carriers.

This mutation (Genbank accession No. KX898029) has not been reported by us in the public databases. Based on the PolyPhen-2 algorithm, the mutation is predicted to be probably damaging with a score of 1.000 [7]. Similarly, in the SIFT (sorting intolerant from tolerant) algorithm, the mutation is predicted to be not tolerated and affect protein function with a score of 0.00 [8].

To investigate the properties of the mutant TBG protein we performed isoelectric focusing (IEF) and $\mathrm{T}_{4}$ binding studies. In the IEF gels it was shown that the isoforms of the mutant TBG were preserved while the concentration (as expressed by the intensity of signal on autoradiography) was clearly reduced. As expected, the degree of signal reduction was greater in the hemizygous male (I-1 of family 1) than in the heterozygous female (II-2 of family 1; Fig. 1d). This contrasts with mutations that affect the TBG charge or glycosylation and, thus, the IEF mobility [9].

Furthermore, the affinity of the mutant TBG molecule for $\mathrm{T}_{4}$ was evaluated with binding studies in transfected Cos-7 cells expressing either the normal TBG or the V215G variant. Compared to the normal TBG, the mutant TBG V215G demonstrated significantly decreased binding for $\mathrm{T}_{4}$, of on average $31.1 \pm 2.2 \%$.

\section{Discussion}

We report a novel TBG variant, V215G, or TBG-Glencoe, associated with TBG-PD, identified in 2 unrelated families. Interestingly, both families were of Ashkenazi Jewish origin whose ancestors came from Russia, which suggests that they may share a common progenitor.
Variants causing TBG-PD may have reduced affinity to bind thyroid hormones, reduced stability or abnormal migration patterns on IEF. In variants with lower affinity for $\mathrm{T}_{4}$ there is a discrepancy between the greatly reduced total serum TH concentration relative to the corresponding TBG levels [1]. In the in vitro experiment using Cos-7 cells transfected with either normal or the mutant TBG, it was demonstrated that the $\mathrm{V} 215 \mathrm{G}$ variant has reduced binding affinity for $\mathrm{T}_{4}$. This is in line with in silico data from the 2 algorithms (PolyPhen-2 and SIFT) predicting that the $\mathrm{V} 215 \mathrm{G}$ mutation is expected to significantly alter the function of TBG protein. The results of IEF indicated that the mutant molecule retains the normal TBG isoforms; however, the concentration, as expressed by signal intensity, was lower in the samples of affected subjects compared to normal individuals. In the IEF gel, a difference in signal intensity could be observed between a hemizygous male and a heterozygous female, corresponding to the differences in serum TBG concentration.

In family 1 , the proposita presented with delayed puberty and altered TFTs. She was given treatment with levothyroxine and, despite TSH normalization, her $\mathrm{TT}_{4}$ values remained low. She presumably had concurrent autoimmune hypothyroidism, possibly transient, which explains why her TSH was not further suppressed and no thyrotoxic symptoms developed. The course of her TFTs while on $\mathrm{L}_{-} \mathrm{T}_{4}$, as well as the TFT alterations in other family members, pointed to a diagnosis of TBG-PD. If left undiagnosed, the patient would have been at risk for continuing unnecessary L- $\mathrm{T}_{4}$ treatment with deleterious effects, especially on her postpubertal bone accrual. In family 2 , the proband had a history of a stable, nonsecretory pituitary adenoma affecting only the gonadotroph function. The timely diagnosis of TBG-PD prevented the initiation of $\mathrm{L}_{-} \mathrm{T}_{4}$ treatment for presumed secondary hypothyroidism, which at his age of 71 years would have considerably increased his risk for cardiac arrhythmias and bone loss.

These 2 cases illustrate the importance of clinical awareness to correctly identify individuals with TBG abnormalities, because their TFT alterations may be misinterpreted and lead to unwanted treatment. Confirmation of the mutation with genetic analysis also allows for the identification of carriers within the family and appropriate genetic counseling.

In conclusion, we have reported a novel TBG variant, V215G or TBG-Glencoe, causing TBG-PD in 2 unrelated families of Ashkenazi Jewish origin. The biochemical, in silico and in vitro data collectively indicated that the mutant TBG protein has decreased binding for $\mathrm{T}_{4}$. 


\section{Acknowledgments}

This work was supported by grant R37DK15070 from the National Institutes of Health to S.R. The content is solely the responsibility of the authors and does not necessarily represent the official views of the National Institute of Diabetes and Digestive and Kidney Diseases or the National Institutes of Health.

\section{Disclosure Statement}

The authors have no conflicts of interest to disclose.

\section{References}

1 Bartalena L: Recent achievements in studies on thyroid hormone-binding proteins. Endocr Rev 1990;11:47-64.

$>2$ Hayashi Y, Mori Y, Janssen OE, Sunthornthepvarakul T, Weiss RE, Takeda K, Weinberg M, Seo H, Bell GI, Refetoff S: Human thyroxine-binding globulin gene: complete sequence and transcriptional regulation. $\mathrm{Mol}$ Endocrinol 1993;7:1049-1060.

-3 Pappa T, Ferrara AM, Refetoff S: Inherited defects of thyroxine-binding proteins. Best Pract Res Clin Endocrinol Metab 2015;29: 735-747.
4 Takamatsu J, Refetoff S, Charbonneau M, Dussault JH: Two new inherited defects of the thyroxine-binding globulin (TBG) molecule presenting as partial TBG deficiency. J Clin Invest 1987;79:833-840.

5 Mannavola D, Vannucchi G, Fugazzola L, Cirello V, Campi I, Radetti G, Persani L, Refetoff S, Beck-Peccoz P: TBG deficiency: description of two novel mutations associated with complete TBG deficiency and review of the literature. J Mol Med (Berlin) 2006;84:864-871.

6 Kambe F, Seo H, Mori Y, Murata Y, Janssen OE, Refetoff S, Matsui N: An additional carbohydrate chain in the variant thyroxinebinding globulin-Gary (TBGAsn-96) impairs its secretion. Mol Endocrinol 1992;6:443449.
Ramensky V, Bork P, Sunyaev S: Human nonsynonymous SNPs: server and survey. Nucleic Acids Res 2002;30:3894-3900.

8 Kumar P, Henikoff S, Ng PC: Predicting the effects of coding non-synonymous variants on protein function using the SIFT algorithm. Nat Protoc 2009;4:1073-1081.

9 Mori Y, Seino S, Takeda K, Flink IL, Murata Y, Bell GI, Refetoff S: A mutation causing reduced biological activity and stability of thyroxine-binding globulin probably as a result of abnormal glycosylation of the molecule. Mol Endocrinol 1989;3:575-579. 